\title{
A Scrotal Arteriovenous Malformation: A Case Report
}

\author{
Nobuhiro Sato, Tomoaki Kuroki, Yasuyoshi Tosa, Taro Kusano, Shinya Yoshimoto
}

Department of Plastic and Reconstructive Surgery, Showa University Hospital, Tokyo, Japan.

Email: hotn310@hotmail.com

Received December $23^{\text {rd }}, 2013$; revised January $14^{\text {th }}, 2014$; accepted January $21^{\text {st }}, 2014$

Copyright (c) 2014 Nobuhiro Sato et al. This is an open access article distributed under the Creative Commons Attribution License, which permits unrestricted use, distribution, and reproduction in any medium, provided the original work is properly cited. In accordance of the Creative Commons Attribution License all Copyrights (c) 2014 are reserved for SCIRP and the owner of the intellectual property Nobuhiro Sato et al. All Copyright @ 2014 are guarded by law and by SCIRP as a guardian.

\section{ABSTRACT}

Purpose: Haemangiomas and vascular malformations are not easy to differentiate clinically. We describe a patient who was initially thought to have a scrotal venous malformation (VM) but after pathological examination was diagnosed to be afflicted with an arteriovenous malformation (AVM) with a micro-fistula in pathorogical photo. We think that even if there are suspicions of VM before the operation, the importance of resection with an ample margin of safety is indicated. Material and Method: A 38-year-old man who noticed a mass in his scrotum. The patient was seen at our hospital in May 2009 with concern for progression of the scrotal mass. That mass appeared to indicate a simple case of VM of the scrotum. Result and Conclusion: A biopsy of the mass was performed and was diagnosed to be a micro-fistula AVM, with no tumour growth in the vascular endothelial cells. Resection was performed in September 2009. And there is no recurrence. Even if there are suspicions of VM before the operation, the importance of resection with an ample margin of safety is indicated.

\section{KEYWORDS}

\section{Arteriovenous Malformation; Scrotum; Resection}

\section{Introduction}

There are two types in vascular anomalies, one shows endothelial hyperplasia designated as hemangiomas, and the other, which does not show endothelial hyperplasia, is called malformation. We report a patient who was initially believed to have a scrotally-positioned venous malformation (VM) before the operation and later diagnosed as an arteriovenous malformation (AVM) upon biopsy and pathological analysis. A complete resection was performed of the lesion. Haemangiomas and vascular malformations are not easy to differentiate clinically and even if there are suspicions of VM before the operation, the importance of resection with an ample margin of safety is indicated (Table 1).

\section{Material and Method}

The patient is a 38-year-old man who at the age of 18 noticed a mass in his scrotum and had undergone removal of part of the mass at another hospital. The patient, however, was not able to provide the diagnosis of the
Table 1. The abbreviations list in this article.

\begin{tabular}{cc}
\hline VM & Venous malformation \\
AVM & Arteriovenous malformation \\
AVF & Arteriovenous fistula \\
\hline
\end{tabular}

mass from this first operation. There was no notable previous history including any history of genital trauma. The patient was seen at our hospital in May 2009 with concern for progression of the mass because it had increased in size postoperatively. On visual inspection a $10-\mathrm{mm}$ wide protrusion was observed from the centre of the scrotum on the anal side extending past the penis to the frenulum of the glans penis (Figure 1). Upon palpation, the mass was found to be elastic, soft and tuberous, ranging from bluish-purple to brown in colour. The mass was without any appreciable pulsation and the patient endorsed no external bleeding or bloody urine. These observations appeared to indicate a simple case of VM of the scrotum. Contrast CT was performed demonstrating a high-signal area corresponding to the mass region on the 


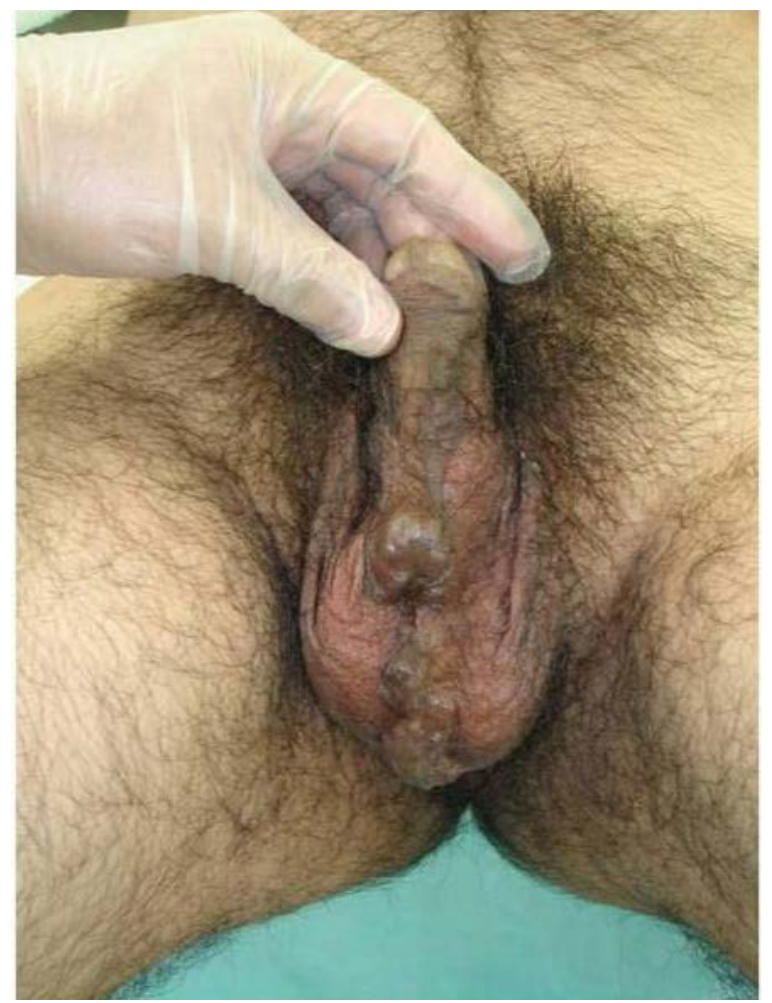

Figure 1. On visual examination a 10-mm wide protrusion was observed from the centre of the scrotum on the anal side meandering past the penis to the frenulum of glans.

venous phase images and no imaging results in the arterial phase, indicating a genitally-localised VM. A biopsy of the mass was performed in July 2009. Pathological examination of the mass with Elastica van Gieson staining demonstrated that the intima and media in part of the vein were dilated and an area that appeared to be arterial in nature, associated with an arterial anastomosis (Figure 2). The mass was diagnosed to be a micro-fistula AVM, with no tumour growth in the vascular endothelial cells. Operation was planned for complete resection of this localised lesion.

\section{Result}

Resection was performed in September 2009 under lumbar spinal anaesthesia. A zigzag skin incision was made from the centre of the scrotum on the anal side to the frenulum of the glans penis. The vascular tissue was limited to the dermis and tunica dartos layer, and resection included surrounding normal tissue such that the mass itself was not damaged. Swelling was marked in both the proximal and distal regions of the biopsied site and the skin overlying the adherent scar was excised (Figure 3). In December 2010, 15 months postoperatively, a contrast CT was performed showing absence of the high-signal region that was present before the operation and additional physical examination revealed that there was no

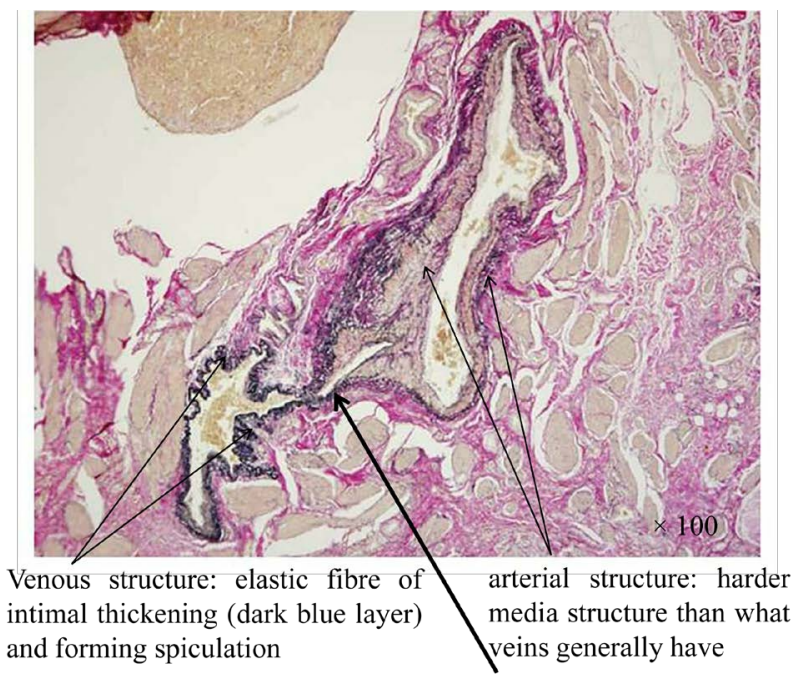

Anastomosis was observed on both sides.

Figure 2. On Elastica van Gieson staining $(\times 100)$, on the left side dark blue layer indicates elastic fibre of intimal thickening and forming speculation. There was a venous structure through increase in venous pressure. A swelling structure in the intima and media in the cohering elastic fibre of the vessels on the right side was observed and it was determined to be an artery with a harder media structure than what veins generally have. Anastamosis was observed on both sides (arrow). It was evidently micro-fistula AVM, with no tumorous growth in the vascular endothelial cells.

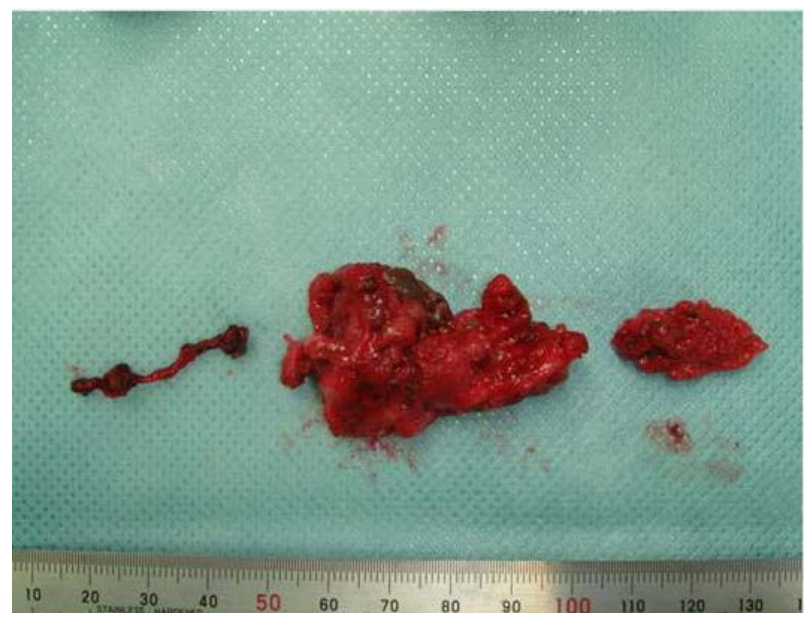

Figure 3. Totally resected specimen. An operation was planned to totally resect the localised lesion.

evidence of recurrence (Figure 4).

\section{Discussion}

AVM and arteriovenous fistula (AVF) are classified as "combined-type high-flow vascular malformations" in The International Society for the Study of Vascular Anomalies classification [1-3]. With the exception of instances in which it is present intraorbitally or in extensive lesions, high-velocity AVM and AVF are treated by re- 


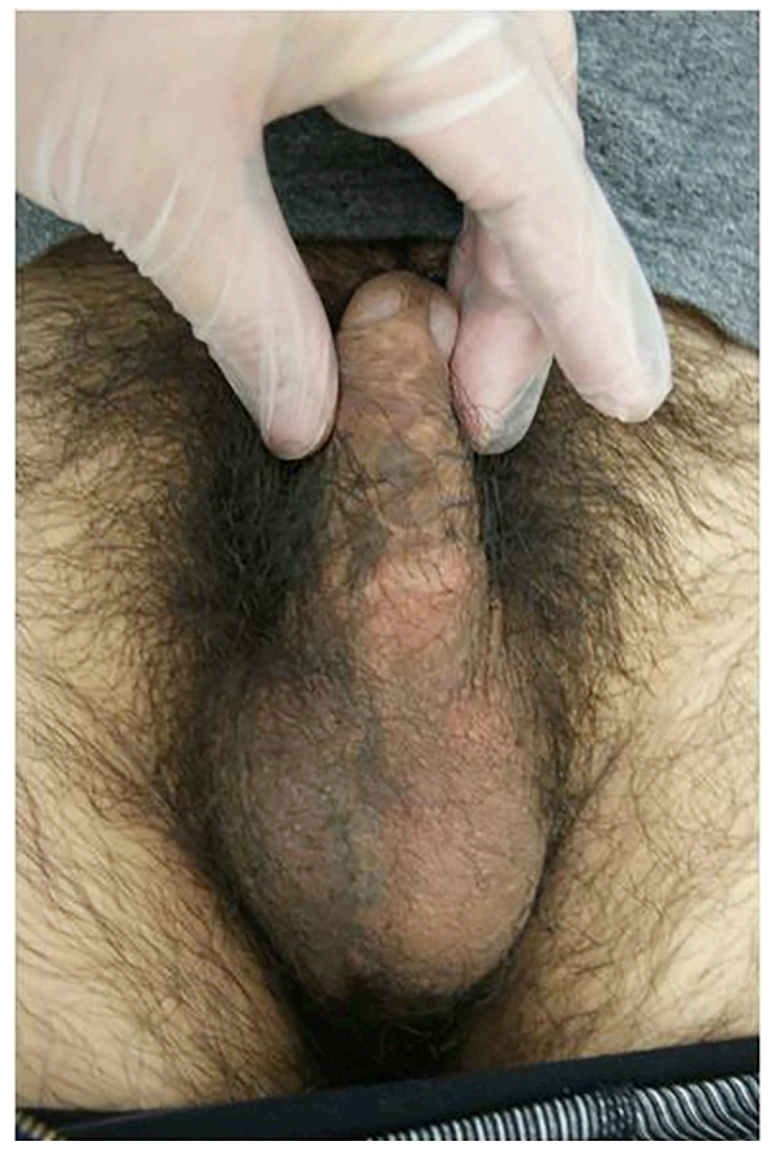

Figure 4. A 15 months post operative photograph showed absence of the tumour that was present before the operation.

section and sclerotherapy or embolisation [4-6]. Laser irradiation does not provide a complete treatment [7], and radiation as well as steroid administration are both said to be ineffective. In this case in which pulsations were not appreciated upon palpation and there were no imaging correlates during the arterial phase of the contrast CT, the malformation was suspected to be a low-flow VM of the scrotum. The defining point of AVM diagnosis is the presence of a nidus seen on arterial imaging. Regarding AVFs, although it is standard to base the diagnosis on arteriovenous shunting, which can be clearly determined by arterial imaging and contrast CT, the presence of some fistulas cannot be confirmed even by microscopic examination. Extended resection is recommended to prevent recurrence from a missed nidus when performing extirpative surgery in low-flow type AVM. In this case, no signs of recurrence were observed 15 months after the operation. For AVM, Stages III and IV of the Schobinger classification predicates an aggressive treatment plan. A wait-and-see approach is commonly employed for stages I and II or paediatric cases, in which there is a gradual progression of the disease. In this case the mass was classified to be stage I. Extremely localised lesions have generally poor results with AVM resection, however, there is also a school of thought that maintains that resection can produce good results for stage I localised forms $[8,9]$. Because of the rapid progression of the disease in this patient as well as the desire and motivation of the patient, operation was offered as a treatment strategy. In terms of the pathological findings, on the left side there was a venous structure of cohesive elastic fibrous tissue, forming spiculations through anincrease in venous pressure. A swollen structure in the intima and media of the elastic fibres of the vessels on the right side was observed and this was determined to be an artery because of the harder medial structures than that demonstrated in normal venous tissue. Arterial-venous anastomosis was observed on both sides. Fistulas have been classified into three types by angiography in a report by Houdart et al. [10], and a portion of these structures was identified in this patient. In addition, according to the Houdart report, in cases of cerebral AVF there is an approximately 5\% incidence of malformation in which a feeder artery connects to a direct emissary vein without an interjacent nidus. By the presence of this type of AVF, it is possible to determine at pathological examination that a given structure initially appearing to be AVM is actually an AVM.

\section{Conclusion}

Haemangiomas and VM are not easy to differentiate clinically and even if there are suspicions of VM before the operation, the importance of resection with an ample margin of safety is indicated.

\section{REFERENCES}

[1] J. B. Mulliken and J. Glowacki, "Hemangiomas and Vascular Malformations in Infants and Children: A Classification Based on Endothelial Characteristics,” Plastic and Reconstructive Surgery, Vol. 69, No. 3, 1982, pp. 412420. http://dx.doi.org/10.1097/00006534-198203000-00002

[2] O. Enjolras and J. B. Mulliken, "Vascular Tumors and Vascular Malformations (New Issues),” Advances in Dermatology, Vol. 13, 1998, pp. 375-423.

[3] O. Enjolras, R. Chapot and J. J. Merland, "Vascular Anomalies and the Growth of Limbs: A Review," Journal of Pediatric Orthopaedics B, Vol. 13, No. 6, 2004, pp. 349357.

http://dx.doi.org/10.1097/01202412-200411000-00001

[4] K. Ouchi, T. Wakita and S. Wakita, "Direct Puncture Sclerotherapy for Arteriovenous Malformation," Japanese Journal of Interventional Radiology, Vol. 18, 2003, pp. 139-143.

[5] T. Nomura, A. Sakurai, I. Nagata, H. Terashi and S. Tahara, "Our Strategy for the Treatment of Venous Malformations,” Japanese Journal of Phlebology, Vol. 19, No. 3, 2008, pp. 161-168. 
[6] J. Upton III and J. J. Marler, "Vascular Anomalies of the Upper Extremity,” In: S. J. Mathes, Ed., Plastic Surgery, Vol. 8, 2nd Edition, Saunders Elsevier, Philadelphia, 2006, pp. 369-416.

[7] S. Watanabe, "Laser Treatment in Vascular Tumors and Malformations," Japanese Journal of Interventional Radiology, Vol. 21, 2006, pp. 390-392.

[8] J. J. Marler and J. B. Mulliken, "Vascular Anomalies,” In: S. J. Mathes, Ed., Plastic Surgery, Vol. 5, 2nd Edition, Saunders Elsevier, Philadelphia, 2006, pp. 19-68.
[9] J. Minakuchi, K. Takahashi, T. Okamura, et al., “A Case of Congenital Scrotal a-v Fistula,” Japanese Journal of Clinical Urology, Vol. 38, 1984, pp. 353-355.

[10] E. Houdart, Y. P. Gobin, A. Casasco, A. Aymard, D. Herbreteau and J. J. Merland, "A Proposed Angiographic Classification of Intracranial Arteriovenous Fistulae and Malformations,” Journal of Neuroradiology, Vol. 35, No. 5, 1993, pp. 381-385. http://dx.doi.org/10.1007/BF00588376 